\section{Permeability of hyperosmotic agent in normal and atherosclerotic vascular tissues}

\author{
Mohamad G. Ghosn, ${ }^{a}$ Esteban F. Carbajal, ${ }^{a}$ \\ Natasha A. Befrui, ${ }^{\text {a }}$ Armando Tellez, Juan F. Granada, ${ }^{\text {c }}$ \\ and Kirill V. Larin ${ }^{\text {a,* }}$ \\ ${ }^{a}$ The University of Houston, Biomedical Engineering \\ Program, Houston, Texas 77204 \\ ${ }^{\mathrm{b}}$ The Methodist Hospital, The Methodist DeBakey Heart \\ Center, Houston, Texas 77030 \\ ${ }^{\mathrm{C}}$ The Skirball Center for Cardiovascular Research, \\ Cardiovascular Research Foundation, New York, New York \\ 10022
}

Abstract. Noninvasive cardiovascular imaging could lead to the early detection and timely treatment of complex atherosclerotic lesions responsible for major cardiovascular events. Recent investigations have suggested that optical coherence tomography (OCT) is an ideal diagnostic tool due to the high resolution this technology achieves in discriminating the different features of atherosclerotic lesions based on structural imaging. We explore the capability of OCT for functional imaging of normal and atherosclerotic aortic tissues based on time- and depth-resolved quantification of the permeability of biomolecules through these tissues. The permeability coefficient of $20 \%$ aqueous solution of glucose was found to be $(6.80 \pm 0.18)$ $\times 10^{-6} \mathrm{~cm} / \mathrm{s}$ in normal aortas and $(2.69 \pm 0.42)$ $\times 10^{-5} \mathrm{~cm} / \mathrm{s}$ in aortas with atherosclerotic disease. The results suggest that this new OCT functional imaging method-the assessment of the permeability coefficients of various physiologically neutral biomolecules in vascular tissues-could assist in early diagnosing and detecting the different components of atherosclerotic lesions. () 2008 Society of Photo-Optical Instrumentation Engineers. [DOI: 10.1117/1.2870153]

Keywords: atherosclerosis; functional imaging; optical coherence tomography; diffusion.

Paper 07334LR received Aug. 16, 2007; revised manuscript received Nov. 9, 2007; accepted for publication Dec. 9, 2007; published online Feb. 28, 2008.

\section{Introduction}

Accumulation of low-density lipoproteins (LDLs) and other lipid-bearing materials, calcium, and other blood substances in the arterial wall leads to arteriosclerotic disease that is the leading cause of death and disability worldwide. Significant efforts are currently being made to understand, prevent, and cure this disease by many scientists and clinicians around the world. However, despite all efforts, we still do not completely understand what causes this disease and are unable to significantly prevent the occurrence of adverse cardiovascular events.

The aorta is the primary artery in the circulatory system and provides oxygenated blood to all other arteries of the

*E-mail: klarin@uh.edu body except those found in the lungs. The aorta is composed of three different layers: the intima, the media, and the adventitia. The intima is the innermost layer and is almost completely made up of smooth endothelial cells with variable amounts of elastic fibers. The intima includes the endothelial lining and an underlying layer of connective tissue. Past the intima is the media, which is composed of concentric sheets of smooth muscle cells and elastic tissue. The layer farthest from blood flow (the outermost layer) is the adventitia. It is a very thick layer composed mainly of collagen fibers as well as connective tissue and elastic fibers.

Cardiovascular imaging could help in the diagnosis of a variety of conditions. Several imaging techniques have been utilized to accomplish this task. Magnetic resonance imaging (MRI) is commonly used in noninvasive cardiovascular imaging but is very costly, while the frequent application of $\mathrm{x}$ rays may expose the patient to excessive harmful radiation. Intravascular ultrasound is a widely used technique for cardiovascular imaging because of its ability to visualize normal and diseased blood vessels from the inside, ${ }^{1}$ but it has limited resolution, low contrast, and is designed for structural imaging only. Optical coherence tomography (OCT) utilization in minimally invasive intravascular cardiovascular imaging, on the other hand, can offer safe high-resolution images and relatively low cost and can provide vital information to the treating physician. ${ }^{2-5}$

Alterations in the morphology of biological tissues can be caused by different diseases. The alterations in the organization of cells and tissues that compose the aorta could impact the permeability coefficient of solutions and drugs that diffuse through. Therefore, knowledge of diffusion values could aid in diagnosing diseases and help differentiate among normal and abnormal tissues. Recently we demonstrated the capability of OCT for noninvasive and nondestructive monitoring and quantifying of the permeability coefficient of different analytes and drugs in various biological tissues. ${ }^{6-9}$ In this letter, we report results on quantification of diffusion of biomolecules in normal and diseased areas of pig aorta. The diagnosis from information provided by OCT could help in timely and effective treatment.

\section{Materials and Methods}

A time-domain OCT system was employed in the diffusion experiments. Low-coherence broadband light source (Superlum, Inc., Russia) with a wavelength of $1310 \pm 15 \mathrm{~nm}$ and an output power of $3 \mathrm{~mW}$ was used as the optical source. Imaging was continuous with a two-dimensional (2-D) image measuring $2.2 \times 2.4 \mathrm{~mm}$ being acquired every $3 \mathrm{~s}$ by scanning the sample surface laterally with the incident beam and in depth with the interferometer. The estimated resolution of the system was $25 \mu \mathrm{m}$ in air. A single one-dimensional (1-D) curve displaying the distribution of light in depth (on the logarithmic scale) was created by averaging 2-D images laterally (over approximately $1 \mathrm{~mm}$, which was enough for speckle noise suppression).

Aortic tissues harvested from hypercholesterolemic pigs were surgically removed and placed in $0.9 \%$ saline solution. The experiments were performed within $2 \mathrm{~h}$ after extraction. All samples were taken from the aorta of a single pig. A

1083-3668/2008/13(1)/010505/3/\$25.00 @ 2008 SPIE 


\section{JBO LETTERS}

trained pathologist indicated the locations in the tissue that were and were not affected by atherosclerosis by visual examination prior to the OCT experiments. The aortas were cut sagittally to create a sheet, which was then cut to approximately $1-\mathrm{cm}^{2}$-square samples with and without arteriosclerosis. The tissue was submerged in $3 \mathrm{ml}$ of saline solution (to prevent tissue dehydration during the experiments). Continuous imaging was performed for approximately $1 \mathrm{~h}$. About 7 to $9 \mathrm{~min}$ into imaging (required to record a baseline), $3 \mathrm{ml}$ of $40 \%$ glucose solution was added to the saline bath, creating a total volume of $6 \mathrm{ml}$ of $20 \%$ concentrated glucose.

The permeability coefficient of glucose in the aortic tissue was calculated with the OCT signal slope (OCTSS) method, which is explained in details in Ref. 6. The average permeability coefficient was computed by analyzing the OCTSS changes induced by glucose diffusion in a specific depth region. A linear region with minimal fluctuations from the 1-D OCT signal was chosen, and its thickness $\left(z_{\text {region }}\right)$ was calculated. The local increase of glucose concentration in the chosen region caused the scattering coefficient to decrease, which was clearly reflected in the OCTSS graph. The time $\left(t_{\text {region }}\right)$ was calculated by measuring from the point where the OCTSS started to decrease to the point at which a change in the slope of the OCT signal indicated that the diffusion has ended and the reverse process began to take place. An increase of the local glucose concentration induced a decrease of the OCT signal slope. The reverse process, designated by the positive slope change, is thought to have stemmed from the difference in the concentration gradients on both sides of the tissue causing the fluid to travel from the region of higher concentration to the lower one (water reentering the tissue after diffusing out due to the application of glucose). The permeability coefficient $(\bar{P})$ was then calculated by dividing the thickness of the region selected by the time it took for glucose to diffuse through $\left(\bar{P}=z_{\text {region }} / t_{\text {region }}\right)$.

\section{Results and Discussion}

Figures 1(a) and 1(b) are the 2-D OCT images of a normal pig aortic tissue and an aortic tissue with early atherosclerosis, respectively. Typical results obtained from the hematoxyen and eosin (H\&E) histological examination of aortas of the pig model are shown in Figs. 1(c) and 1(d). A vertical view of normal tissues [Fig. 1(c)] shows that the layers look compact and have a normal distribution (note that the endothelium is in one single cell layer, as it should be). In comparison, Fig. 1(d), which represents the thinnest sample from diseased sections, can be seen with significant neointimal thickening.

Figure 2 represents a typical OCTSS graph for a normal pig aorta during a glucose diffusion experiment. The sample was monitored for approximately $9 \mathrm{~min}$ before application of the glucose solution. The monitored region was about $70 \mu \mathrm{m}$ in thickness and $157 \mu \mathrm{m}$ away from the surface. The OCT signal slope decreased due to the reduction of scattering within the tissue caused by the local increase of glucose concentration. Glucose solution reached the monitored region approximately $20 \mathrm{~min}$ after administration and it took another $20 \mathrm{~min}$ for it to completely diffuse through. At that point, a reverse process in the OCTSS was shown. This change in slope could be due to diffusion via concentration gradient differences on either side of the tissue; the net fluid (mainly

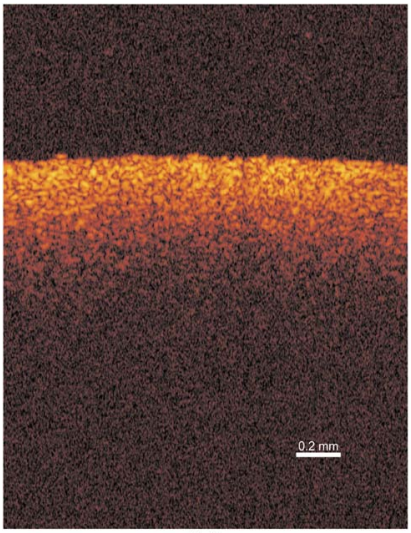

(a)

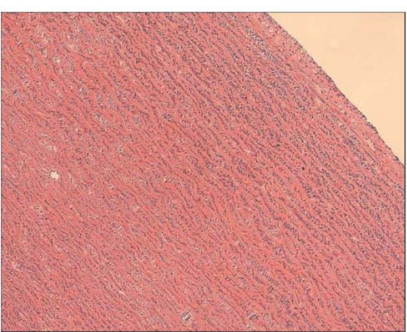

(c)

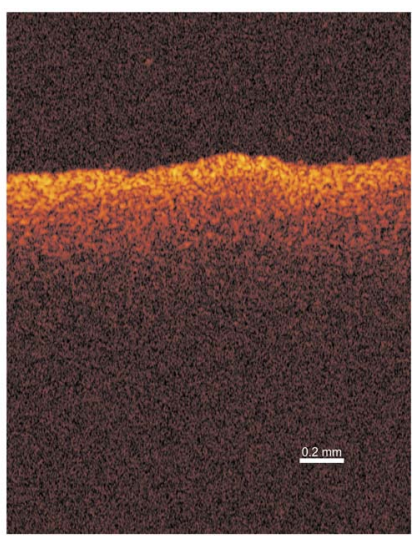

(b)

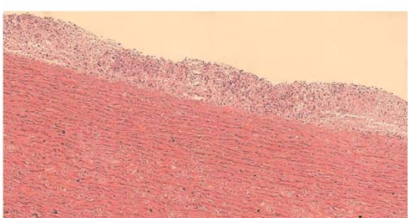

(d)
Fig. 1 (a) and (c) OCT and histology images of a normal pig aorta, respectively, and (b) and (d) OCT and histology images from an early atherosclerotic lesion in a pig aorta.

water) movement from areas of high concentration to those of lower concentration would occur until equilibrium is reached. The permeability coefficient of glucose $20 \%$ from four different experiments was found to be $6.80 \pm 0.18 \times 10^{-6} \mathrm{~cm} / \mathrm{s}$. The uncertainty in the permeability coefficient from five independent readings was $0.07 \times 10^{-6}$.

Figure 3 displays an OCTSS graph during a glucose diffusion experiment in an atherosclerotic pig aorta. The same procedure was implemented as for the normal tissues experiments. The permeability coefficient of glucose $20 \%$ in

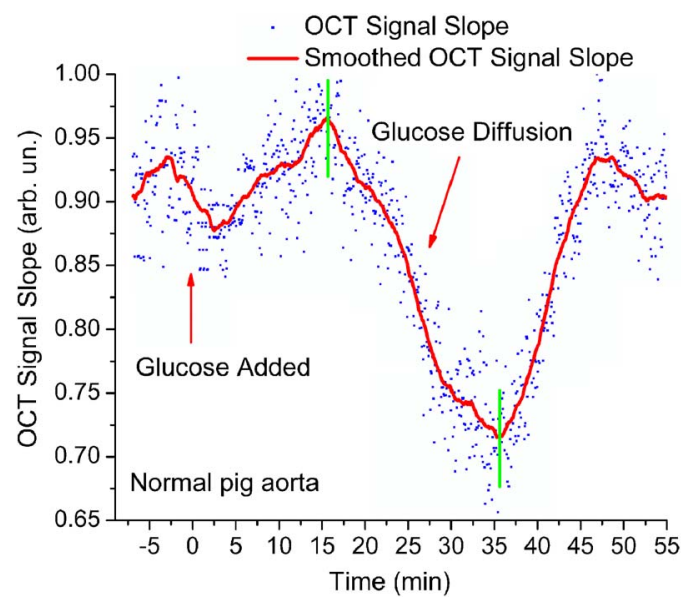

Fig. 2 Typical OCTSS graph as a function of time recorded from a normal pig aorta sample during a glucose $20 \%$ diffusion experiment. The smoothed graph was obtained by averaging intervals on 50 points. The tick marks indicate the interval of glucose diffusion. 


\section{JBO LETTERS}

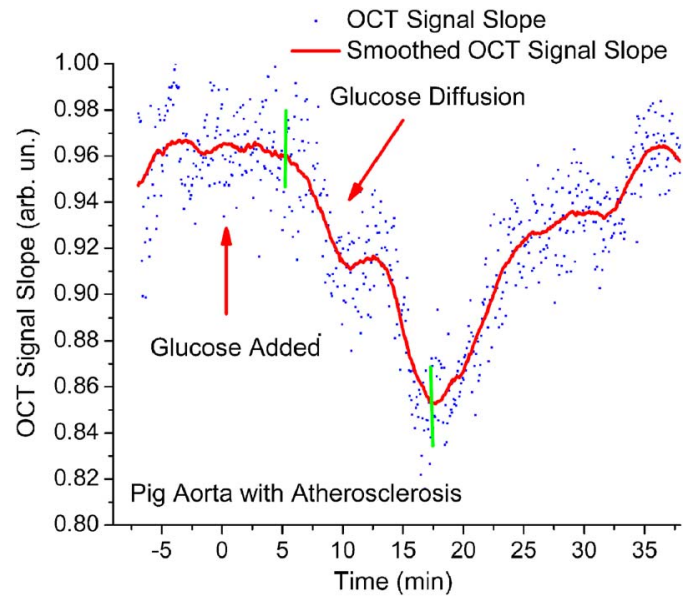

Fig. 3 OCTSS graph as a function of time recorded from an atherosclerotic pig aorta sample during the glucose $20 \%$ diffusion experiment. The smoothed graph was obtained by averaging intervals on 50 points. The tick marks indicate the interval of glucose diffusion.

atherosclerotic aorta tissues was found to be $2.69 \pm 0.42$ $\times 10^{-5} \mathrm{~cm} / \mathrm{s}(n=7)$ with an uncertainty of $0.03 \times 10^{-6}$ from five independent readings.

The results from this study indicate that atherosclerotic tissue had a higher permeability coefficient compared to normal tissue. These findings support the hypotheses that OCT could not only have the capability to measure the permeability coefficient in aortic tissues, but can also distinguish among normal and abnormal regions of the tissues. The increased rate at which glucose diffused into the atherosclerotic tissue could be explained anatomically. LDLs usually diffuse through the different layers of the aortic tissue to reenter circulation but at times can become trapped inside the intima. Inside the intima, the LDLs undergo oxidation and become modified. Macrophages migrate between the endothelial linings of the intima and start taking up the modified LDLs where they become engorged. This can lead to foam cell formation and can eventually lead to an accumulation of plaque that protrudes into the lumen of the aorta. As the atherosclerotic plaque increases in size over time, the elastic tissues that form the aortic layers expand to compensate for the blockage and maintain adequate blood flow throughout the body. The expansion of the aorta could cause the numerous cells, fibers, and tissues of the aorta to readjust their organization. From that organizational change, glucose could find the tissue easier to diffuse through. This expansion could explain the increase in the permeability coefficient of glucose through atherosclerotic tissue.

The structural OCT images from our experiments did not enable effective differentiation of normal from diseased tissues (see Fig. 1). However, the functional analysis showed significant differences between normal and abnormal tissue regions. This information could significantly increase the specificity and accuracy of tissue classification and further the use of OCT in clinical imaging. It is possible that this func- tional imaging method could potentially differentiate components contained in advanced atherosclerotic lesions such as necrotic core and collagen.

\section{Conclusion}

The OCT functional imaging method could supplement existing intravascular structural imaging methods [such as intravascular ultrasound (IVUS) and OCT intravascular structural imaging] and have a significant impact on early diagnostics of vascular abnormalities. These pilot results indicate that structural imaging with OCT may not be able to identify diseased regions; however, functional imaging could enable accurate assessment of analyte diffusion and assist in the early diagnosis of complex atherosclerotic lesions. The permeability coefficient in the abnormal tissues was found to be about four times than that in the normal ones. The results presented could prove the use of OCT in functional cardiovascular imaging and diagnosing as well as detecting atherosclerosis in aortic tissues. While the described proof-of-concept studies measures molecular diffusion over many minutes, it is reasonable to expect that a future clinical utility would not require continuous image acquisition to quantify molecular diffusion in arteries. Additionally, the development or application of other small molecules, various diffusion enhancers, drugs that might be used to treat patients, or even biocompatible nanoparticles might significantly reduce the acquisition/monitoring time, enabling a much faster diagnosis.

\section{References}

1. S. E. Nissen, J. C. Gurley, C. L. Grines, D. C. Booth, R. McClure, M. Berk, C. Fischer, and A. N. DeMaria, "Intravascular ultrasound assessment of lumen size and wall morphology in normal subjects and patients with coronary artery disease," Circulation 84(3), 1087-1099 (1991).

2. S. A. Boppart, G. J. Tearney, B. E. Bouma, M. E. Brezinski, and J. G. Fujimoto, "Noninvasive in vivo assessment of developing cardiovascular morphology and function using high-speed optical coherence tomography," Circulation 94(8), 2828-2828 (1996).

3. B. D. MacNeill, B. E. Bouma, H. Yabushita, L. K. Jang, and G. J. Tearney, "Intravascular optical coherence tomography: cellular imaging," J. Nucl. Cardiol. 12(4), 460-465 (2005).

4. F. J. van der Meer, D. J. Faber, D. M. Baraznji Sassoon, M. C Aalders, G. Pasterkamp, and T. G. van Leeuwen, "Localized measurement of optical attenuation coefficients of atherosclerotic plaque constituents by quantitative optical coherence tomography," IEEE Trans. Med. Imaging 24(10), 1369-1376 (2005).

5. G. J. Tearney, I. K. Jang, and B. E. Bouma, "Optical coherence tomography for imaging the vulnerable plaque," J. Biomed. Opt. 11(2), 021002 (2006).

6. M. G. Ghosn, V. V. Tuchin, and K. V. Larin, "Nondestructive quantification of analyte diffusion in cornea and sclera using optical coherence tomography," Invest. Ophthalmol. Visual Sci. 48(6), 27262733 (2007).

7. M. G. Ghosn, V. V. Tuchin, and K. V. Larin, "Depth-resolved monitoring of glucose diffusion in tissues by using optical coherence tomography," Opt. Lett. 31(15), 2314-2316 (2006).

8. K. V. Larin, M. G. Ghosn, S. N. Ivers, A. Tellez, and J. F. Granada, "Quantification of glucose diffusion in arterial tissues by using optical coherence tomography," Laser Phys. Lett. 4(4), 312-317 (2007).

9. K. V. Larin and M. Ghosn, "Influence of experimental conditions on drug diffusion in cornea," Quantum Electron. 36(12), 1083-1088 (2006) 